\title{
A Brief History of the Opioid Epidemic and Strategies for Pain Medicine
}

Mark R. Jones · Omar Viswanath · Jacquelin Peck · Alan D. Kaye •

Jatinder S. Gill · Thomas T. Simopoulos

Received: March 15, 2018 / Published online: April 24, 2018

(C) The Author(s) 2018

\section{ABSTRACT}

The opioid epidemic has resulted from myriad causes and will not be solved by any simple solution. Consequent to a staggering increase in opioid-related deaths in the USA, various governmental inputs and stakeholder strategies have been proposed and implemented with varying success. This article summarizes the history of opioid use and explores the causes for the present day epidemic. Recent trends in opioid-related data demonstrate an almost fourfold increase in overdose deaths from 1999 to 2008. Tragically, opioids claimed over 64,000 lives just last year. Some solutions have undergone legislation, including the limitation of numbers of opioids postsurgery, as well as growing national prevalence of enhanced

Enhanced digital features To view enhanced digital features for this article go to https://doi.org/10.6084/ m9.figshare.6133172.

M. R. Jones $(\bowtie) \cdot$ O. Viswanath · J. S. Gill ·

T. T. Simopoulos

Beth Israel Deaconess Medical Center, Harvard

Medical School, Boston, MA, USA

e-mail: mjones13@bidmc.harvard.edu

J. Peck

Johns Hopkins Medical Center, All Children's

Hospital, St. Petersburg, FL, USA

A. D. Kaye

Louisiana State University Health Science Center,

New Orleans, LA, USA recovery after surgery protocols which focus on reduced postoperative opioid consumption and shortened hospital stays. Stricter prescribing practices and prescription monitoring programs have been instituted in the recent past. Improvement in abuse deterrent strategies which is a major focus of the Food and Drug Administration (FDA) for all opioid preparations will likely play an important role by increasing the safety of these medications. Future potential strategies such as additional legislative policies, public awareness, and physician education are also detailed in this review.

Keywords: Abuse deterrent formulations; Enhanced recovery after surgery; Non-opioid pain treatments; Opioid epidemic; Overdose

\section{INTRODUCTION}

As of October 16, 2017, the US Government declared the opioid epidemic a public health emergency. The medical community, and particularly pain medicine practitioners, have been active participants and fully aware of the development of the current state. Prescription drug monitoring programs (PDMPs) and the National All Schedules Prescription Electronic Reporting Act (NASPER) have effectively contributed to the reduction in opioid prescriptions 
by $8 \%$ and prescription opioid overdose death rates by $12 \%$ [1]. Despite these substantial reductions in opioid prescriptions in the USA, deaths by opioid overdose continue to escalate at alarming rates: 64,000 people died from drug overdoses in 2016; over 42,000 of those were opioid deaths $[2,3]$. This represents a $20 \%$ increase from the total of 52,000 drug overdose fatalities in 2015. Overdoses related to illegally manufactured fentanyl represent the greatest contribution to the increase, accounting for 20,000 deaths in total; heroin accounted for 15,000 deaths; and prescription drugs for less than 15,000. Figures 1 and 2 present the rates of opioid prescriptions and the rate of opioid-related deaths.

Many parties are responsible for the present day epidemic. Well-intentioned efforts by multiple industries, medical specialty associations, and governmental oversight organizations have resulted in our current climate. This manuscript
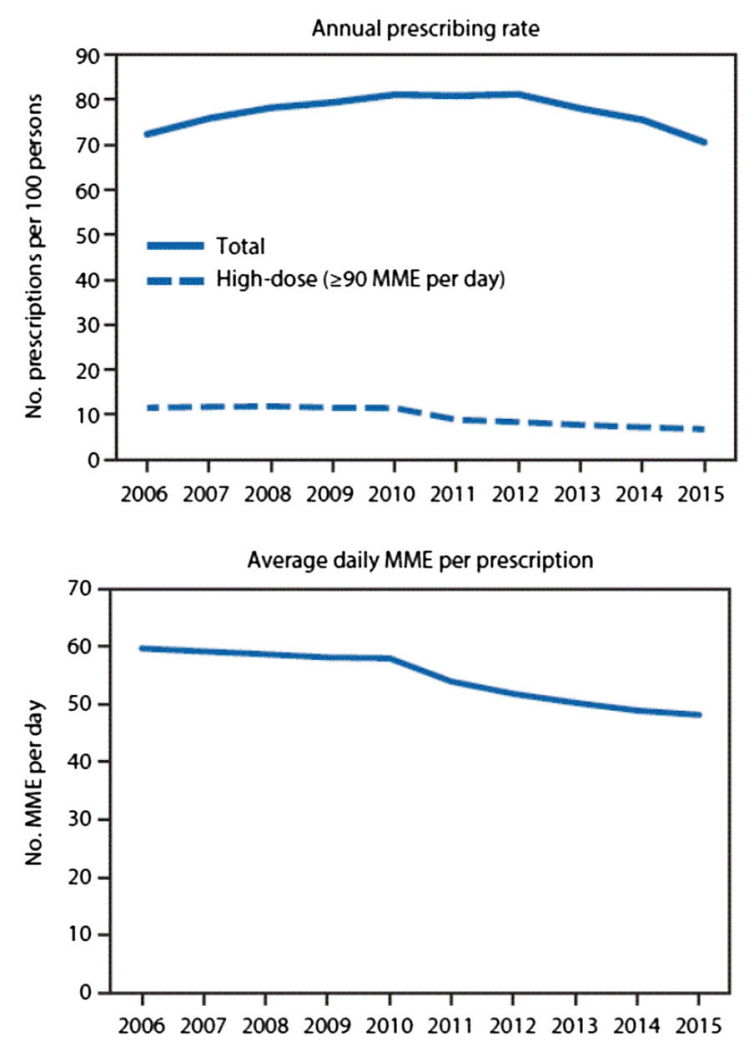

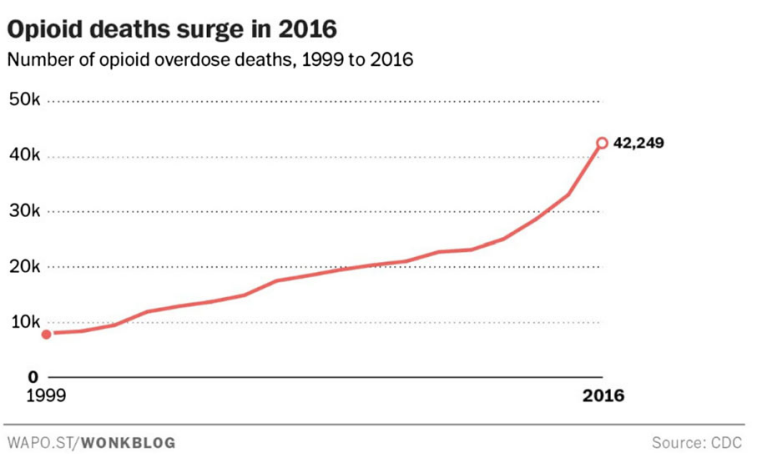

Fig. 2 Number of opioid overdose deaths by category, 1999-2016. Opioid-related deaths increase dramatically in 2016 [5]

will describe a brief overview of the history of opioid therapy as it relates to pain and forecast in detail the ramifications for the practice of pain medicine as an independent and increasingly vital specialty.
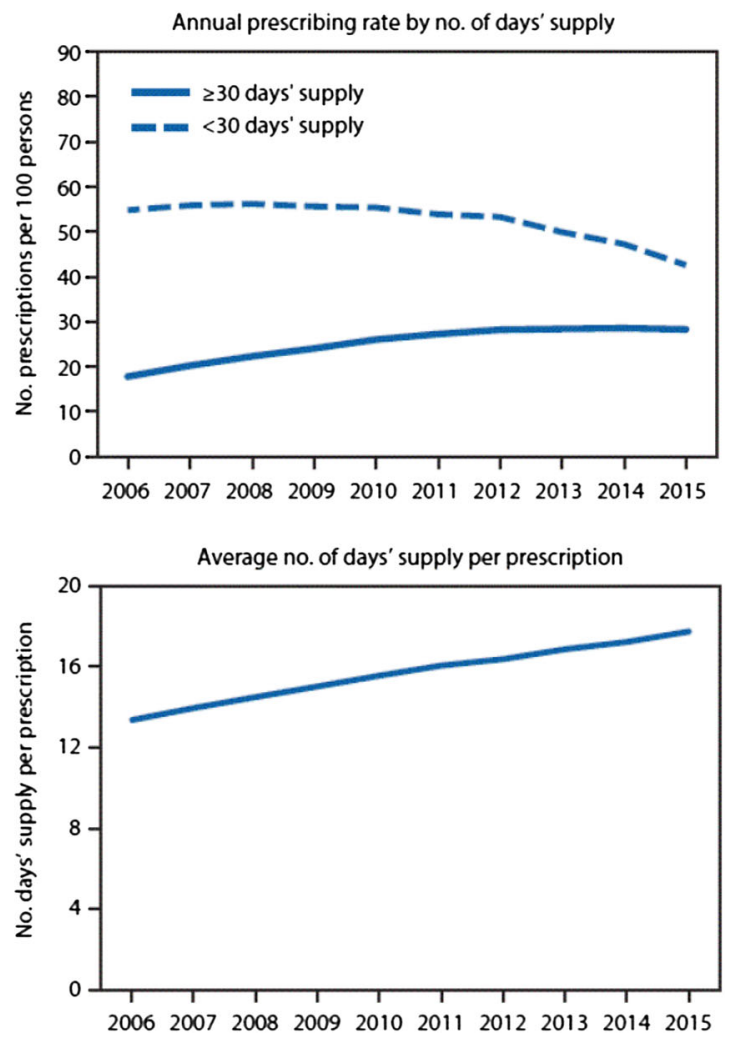

Fig. 1 Annual opioid prescribing rates in the USA, 2006-2015; prescribing rates by number of days' supply; average daily morphine milligram equivalent (MME) per prescription; and average number of days' supply per prescription [4] 


\section{Compliance with Ethics Guidelines}

This article is based on previously conducted studies and does not contain any studies with human participants or animals performed by any of the authors.

\section{BRIEF TIMELINE OF OPIOID ANALGESICS}

Although a detailed description of pain pathways and relevant medical interventions is beyond the scope of this paper, it suffices to say that much of our understanding of pain pathophysiology is recent, and that the field remains in infancy but rapidly evolving. Before 1800 , clinicians regarded pain as an existential phenomenon, a consequence of aging [6]. There was no regulation on the use of cocaine and opioids, resulting in widespread marketing and prescribing for many ailments ranging from diarrhea to toothache [7]. The Harrison Narcotic Control Act of 1914, passed in response to the sudden emergence of street heroin abuse as well as iatrogenic morphine dependence, influenced both physician and patient alike to avoid opiates [8]. Patients with unexplained pain in the 1920s were regarded as deluded, malingering, or abusers, and cancer patients through the 1950s were encouraged to wean themselves off opioids until their lives "could be measured in weeks" [9].

This attitude persisted into the latter half of the twentieth century; a general world-wide "opiophobia" is thoroughly detailed in contemporary literature. Morgan in 1985 and Zenz and Willweber-Strumpf in 1992 both describe a state of under-reliance of opioid analgesics and a resultant under-treatment of pain in Europe and North America [10, 11]. Several developments over this era served to increase awareness of pain under-treatment. A 1973 manuscript from Marks and Sachar in the Annals of Internal Medicine described a failure to treat patients in severe pain with adequate doses of opioid analgesics [12]. Two decades later, Max [13] decried the same failure, invoking the conventional wisdom of the day that "therapeutic use of opiate analgesics rarely results in addiction."
This widespread belief was based upon two small retrospective publications from the 1980s: the first, published as a one paragraph letter to the editor without detailing any scientific rigor, described low $(0.03 \%)$ addiction rates for inpatients receiving opioids for acute pain; the second, a retrospective review of 38 patients, demonstrated that only 2 of 38 patients with chronic pain developed misuse or abuse issues when receiving opioids $[14,15]$. The scientific background for the use of opioids for non-malignant pain was therefore not based upon any demonstrable outcomes or safety studies.

The World Health Organization addressed the under-treatment of postoperative and cancer pain in 1986 with their Cancer Pain Monograph [16]. A rapid improvement in the treatment of cancer pain soon unfurled in many countries, though not entirely, as many countries even today suffer from poor access to opioids [17]. This further prompted a number of publications in the 1990s that questioned the state of pain under-treatment. Notably, Ronald Melzack in 1990 [18] published an article in Scientific American that questioned why opioids were reserved solely for cancer pain and avoided entirely in chronic pain states. The newfound interest bore misconceptions, drawn largely by cancer pain specialists lacking expertise on other chronic, non-cancer pain, that equated the etiologies of malignant and non-malignant pain [15]. This dangerous conflation disregards the complex biopsychosocial phenomena that is chronic pain, and despite many cautions to this effect, opioids grew into the primary modality of chronic non-cancer pain treatment in the USA [19].

Alongside this opioid evolution, the American Pain Society launched their influential "pain as the fifth vital sign" campaign in 1995, with intent to encourage proper, standardized evaluation and treatment of pain symptoms [20]. The Veteran's Health Administration lent support to the campaign with their 1999 adoption of pain as the fifth vital sign initiative [21].

Solidifying the national response to the aforementioned efforts, the Joint Commission (TJC) published standards for pain management in 2000, emphasizing the need for organizations to conduct quantitative assessments of 
pain as recommended by the Institute of Medicine [22]. The Federation of State Medical Boards and the Drug Enforcement Agency also issued statements promising less regulatory scrutiny over opioid prescribers, thereby assuaging physician reluctance to prescribe more liberal amounts of opioid analgesics [23].

The rapid institution of strict standards for pain management in hospital systems culminated in several unintended consequences. Physicians were now mandated to provide adequate pain control by the TJC, resulting in a heavy reliance on opioid medications. The fear among hospital administration was that if new TJC benchmarks were not met, then they were unlikely to receive federal healthcare funds. Indeed, hospitals that invested more readily in opioid therapy generally received better satisfaction rates among their patient population [24]. Pharmaceutical companies heavily pushed the use of opioids as a humane treatment option, often using paid physician consultants to expound on the safety and benefits of opioids use. Not prescribing opioids for a patient with pain risked being labeled as inhumane, often even to the extent of litigation for the undertreatment of pain [25]. Trainees in pain medicine as well as other medical specialties were taught to rely more on opioids for pain treatment. Concurrently, pharmaceutical companies introduced new formulations, such as extended release oxycodone (OxyContin), which were frequently prescribed because of a presumed lower likelihood of abuse, while in reality were heavily abused. From 1997 to 2002, OxyContin prescriptions increased from 670,000 to 6.2 million [26]. Overall opioid consumption continued to climb throughout the 2000s in the USA, rising from $46,946 \mathrm{~kg}$ consumed in the year 2000 to a peak of $165,525 \mathrm{~kg}$ in 2012 [27].

The initial response was favorable, and the push for better pain control appeared successful. One study found that the mean consumption of opiates per patient in the postanesthesia care unit (PACU) increased from $40.4 \mathrm{mg}$ of morphine equivalent to $46.6 \mathrm{mg}$ from 2000 to 2002 with no associated increase in length of stay, naloxone use, or nausea and vomiting [28]. However, concerns soon began to surface regarding overzealous opioid treatment. One report found that the incidence of opioid oversedation more than doubled from 11.0 to 24.5 per 100,000 inpatient hospital days with the implementation of a new standardized, numerical pain treatment algorithm [29]. The Institute for Safe Medication Practices began to establish a link between overaggressive pain management and substantial increases in the incidence of oversedation and associated fatal respiratory depression. The culture change, driven by intent to ensure access to pain relief, had opened the floodgates to the current opioid climate. In just the past 15 years, there has been a proportionate quadrupling of prescription opioid sales and mortality in both men and women based on National Vital Statistics System mortality statistics from the Centers for Disease Control and Prevention [30]. Perplexingly, in addition to the increasing mortality, no study to this date has established level I evidence for the long-term safety and efficacy of opioid therapy in reducing chronic pain intensity and improving function. Instead, numerous other ills arising from opioid medication, such as hyperalgesia, increasing disability, and a host of other formidable problems, including endocrine and psychological co-morbidities, have emerged in relation to chronic opioid use.

It must be noted that pharmaceutical companies contributed significantly to the rise of the opioid epidemic, receiving considerable reprimands as a consequence. In 2007, as the opioid epidemic began to inflict profound damage, Purdue Pharma pleaded guilty to federal charges related to the misbranding of OxyContin. Purdue agreed to pay a total of $\$ 634.5$ million to resolve Justice Department investigations, as well as a $\$ 19.5$ million settlement to 26 states and the District of Columbia [31]. Allegations accuse Purdue of intentionally downplaying the risk of addiction posed by OxyContin and misleading both physicians and the healthcare industry as a whole by overstating the benefits of opioids for chronic pain. At the time of this publication, at least 14 states have submitted lawsuits against the privately held Purdue, and the company has announced that it will cut its sales force in half and stop promoting opioids to physicians in a stark reversal of policy from recent decades. 


\section{REACTION TO OPIOID EPIDEMIC}

In response to the current epidemic, changes in focus to the development of new abuse deterrent opioid formulations at the US Food and Drug Administration (FDA) as well as drafting of new public standards for pain treatment were created at TJC in 2017 [32]. Efforts to develop and provide access to medications intended to treat opioid dependence had begun some time before, however. The Drug Addiction Treatment Act of 2000 (DATA 2000) allowed physicians with a waiver from the Center for Substance Abuse and Mental Health Services Administration to prescribe schedule III, IV, and V medications to treat opioid dependence. The FDA approval in 2002 of buprenorphine and buprenorphine/naloxone formulations allowed specially trained primary care physicians (PCPs) access to these medications. The 2006 Reauthorization Act increased the allowable maximum number of buprenorphine patients per PCP from 30 to 100 patients. These efforts greatly enhanced access to treatment for opioiddependent patients.

In response to the opioid epidemic, FDA public policy changes were announced in February 2016. Among these new positions were a re-examination of the risk-benefit paradigm for opioids with strict emphasis on the large public health ramifications; expanded access to and encouraged development of abuse-deterrent opioid formulations; expert advisory committee assembly prior to new applications for opioids lacking abuse-deterrent properties; improved access to naloxone and other treatment options for opioid use disorder; inclusion of safety information and warnings on immediate-release (IR) opioid labeling; and support for alternative pain management modalities. The various modified opioids released over the past 20 years, such as tamper-resistant preparation, have had differing levels of success, and are collectively referred to as Risk Evaluation and Mitigation Strategies (REMS); these include Targiniq $^{\circledR}$ (oxycodone and naloxone), Suboxone $^{\circledR}$ (buprenorphine/naloxone), reformulated OxyContin $^{\circledR}$ ER (extended release), among others [33-37]. Presently, fewer than one dozen
FDA-approved Abuse-Deterrent Formulations (ADFs) exist, but further development and improvements are ongoing [38].

The January 2017 release of new TJC standards continued the focus on appropriate and effective management of pain, including the recommended inclusion of psychosocial risk factors that may affect self-reporting pain in any pain assessment; set realistic goals when developing treatment plans with patients; emphasize impairment of physical function during pain assessment; emphasize diligent monitoring of opioid prescribing patterns; and promote use of non-pharmacologic pain treatments. In addition, the draft suggests changes to pain management designed to prevent diversion, such as identification of high-risk patients, readily available equipment for monitoring high-risk patients, easy clinician access to prescription monitoring programs databases, and educating patients and families on the safe use, storage, and disposal of opioid medications. Changes have also been put in place at the state level, including providing more limited and standardized doses of opioids for acute pain management and required continuing education for all clinicians who prescribe opioid medications. In this regard, a joint Food and Drug Administration Advisory in 2016 voted unanimously to require continuing education for all opioidprescribing physicians, regardless of specialty, and presently remains a common element of quality improvement projects [39].

\section{IMPLICATIONS FOR PAIN MEDICINE}

With the evolution and manifestation of the current opioid epidemic, opioid prescription and use dependence have gained national attention at the forefront of healthcare politics. Related research and publications in peer-reviewed journals have grown exponentially, and government policy has adapted to meet growing concerns surrounding opioids, including greater scrutiny and laws limiting opioid doses during the first week of acute pain management [40]. Maintaining adequate pain control is still a priority, but efforts to titrate opioid use and to 
increase the use of multimodal pain regimens and ADFs are underway. Enhanced recovery after surgery techniques focus on regimens that include nerve blocks, non-steroidals, gabapentinoids, acetaminophen, and ketamine to decrease postoperative opioid consumption and reduce hospital stays.

As stated earlier, the conflation of cancer pain with chronic non-malignant pain ignored the biopsychosocial complexities underlying the latter, leaving a multidisciplinary approach to the wayside in favor of an overreliance on opioids. Pain management as a specialty will benefit from reinstitution of such multidisciplinary involvement, with emphasis on multimodal analgesia, interventional therapies, and outcomes stressing improvement in physical function.

There is also a growing focus on preventing opioid use disorder (OUD) and on offering affected individuals accessible and effective treatment. US government policy reflects these changes and both the Affordable Care Act and the Mental Health Parity and Addiction Equity Act were major steps forward in treating opioid addiction. The Affordable Care Act, which was signed into law in 2010, with major provisions coming into effect by 2014, dramatically reduced the number of uninsured patients within the USA by 2016 and ensured access to essential health benefits including substance use disorder services and rehabilitative services [41]. This gave opioid-dependent individuals more accessible and affordable routes to treatment. Similarly, the Mental Health Parity and Addictions Equity Act, which took effect in 2010, prevented insurers from placing greater restrictions on mental health and substance abuse disorder treatment benefits than benefits for medical and surgical care [42]. Both initiatives reduce barriers to effective treatment.

Modern culture surrounding opioid use disorder has also changed. Recent studies confirm that treatment for OUD is most effective when comprised of multimodal interventions that are both pharmacological and psychosocial [43]. Therefore, peer-led support groups, communitywide prevention strategies, and stigma-reducing initiatives continue to develop and to demonstrate varying degrees of success in response to the opioid epidemic $[44,45]$. In this regard, one recent publication also addressed the role of local government in the opioid crisis and stressed the importance of local public health coalitions aimed at preventing overdoses and promoting treatment options, as well as citywide anti-stigma education [45].

\section{POTENTIAL STRATEGIES}

Governmental and regulatory agencies' new legislation necessitates a shift in tactics by medical specialty associations in order to comply with and respond to the new perspective. It is crucial that the response to the opioid epidemic does not eradicate the significant strides made over the past half century in our understanding of pain and the enrichment of the therapeutic arsenal at our disposal. This is reflected by the 2010 Declaration of Montreal released by the International Association for the Study of Pain, wherein they maintain and reemphasize the ideal that the relief of suffering and pain is a moral duty of physicians, and that access to pain treatment is a fundamental human right [46]. Following this light, in order to continue to curb opioid abuse and reduce opioid deaths while maintaining adequate access to pain relief, the American Society of Interventional Pain Physicians (ASIPP) has issued recommendations focused on two main arenas: more effective legislative efforts while maintaining appropriate access, and the promotion of non-opioid modalities including interventional techniques. These recommendations are comprised in three separate tiers.

Tier 1 stresses aggressive public education to explicitly address the dangers of illicit drugs (heroin and fentanyl); the adverse consequences of opioid abuse along with the increased risk of opioids combined with benzodiazepines; mandatory $4 \mathrm{~h}$ of continuing physician education per year for all prescribers of any amount of opioid or benzodiazepine; and mandatory patient education associated with the first prescription of any amount of opioid. According to a recent survey published in the New England Journal of Medicine, a significant percentage of the public blame the opioid 
epidemic on doctors who inappropriately prescribe medication $(46 \%)$, with only $28 \%$ allocating blame to people who illegally sell prescription pain killers, and $13 \%$ blaming pharmaceutical companies [47]. In this same survey, the public believes public awareness and education programs are effective and should be continued.

ASIPP's tier 2 recommendations emphasize ease of access along with lower or no copayments for directed modalities based on non-opioid pain therapy including physical therapy and interventional techniques. Interestingly, evidence demonstrates a direct correlation between the decline in the utilization of interventional techniques since 2010 secondary to decreasing reimbursements and the ultimate escalation in opioid deaths over the same period [48]. Until prior authorizations for non-opioid treatment modalities are eliminated, and prior authorizations for opioid treatments increased, the arsenal of nonopioid therapies available to pain medicine clinicians will be limited, and the reliance on opioids will continue. Another strategy emphasized in tier 2 is the expansion of low-threshold access to buprenorphine for opioid use disorder, which, after implementation in France, effectively decreased opioid overdose deaths by $79 \%$ over a 6-year period [49]. Lastly, tier 2 recommendations include the establishment of prescription drug monitoring programs (PDMP) that are capable of interstate communication, as well as mandatory provider review of PDMP data prior to prescribing.

The third tier of ASIPP's approach focuses on buprenorphine and methadone. ASIPP recommends that buprenorphine be changed to a schedule II substance, and be made mandatorily available for chronic pain management in addition to medication-assisted treatment. Finally, ASIPP recommends in tier 3 that methadone be removed from the formulary, as it is responsible for over 3000 deaths per year despite comprising in total only $1 \%$ of prescribed opioids.

\section{CONCLUSION}

The opioid epidemic has arisen in part from a complex history of well-intentioned efforts by multiple industries to balance adequate and vital treatment of pain against the misuse and abuse of opioid medications. The presumption that opioids are safe and effective for chronic pain over the previous decade led to the overreliance on opioids and a significant reduction in the number of multidisciplinary pain centers across the USA that were proven effective for this condition. A reduction in reimbursement for such multispecialty centers further shifted management in the direction of pharmacotherapy. Nevertheless, the current state of rampant abuse and consequential fatal overdoses afflicting the USA is unacceptable and deserving of a profound response. There is a clear need to end the focus on blaming responsible parties and shift to implementing solutions. To that end, governmental regulatory agencies and specialty-specific physician associations have released multiple regulatory measures and societal recommendations that together have contributed to a decrease in the amount of opioid prescriptions over recent years. Concerted efforts from multiple disciplines, including physicians, legislators, pharmaceutical companies, educators, and the general public are required to ensure that the epidemic does not continue into the future.

\section{ACKNOWLEDGEMENTS}

Funding. No funding or sponsorship was received for this study or publication of this article.

Authorship. All named authors meet the International Committee of Medical Journal Editors (ICMJE) criteria for authorship for this article, take responsibility for the integrity of the work as a whole, and have given their approval for this version to be published.

Disclosures. Mark R. Jones, Omar Viswanath, Jacquelin Peck, Jatinder S. Gill, and Thomas T. Simopoulos have nothing to disclose. Alan D. Kaye is on the Speakers Bureau for Depomed, Inc. and Merck, and has served on the FDA Advisory Board on Analgesics, Anesthetics, and Addiction Medicine since 2012. 
Compliance with Ethics Guidelines. This article is based on previously conducted studies and does not contain any studies with human participants or animals performed by any of the authors.

Open Access. This article is distributed under the terms of the Creative Commons Attribution-NonCommercial 4.0 International License (http://creativecommons.org/licenses/ by-nc/4.0/), which permits any noncommercial use, distribution, and reproduction in any medium, provided you give appropriate credit to the original author(s) and the source, provide a link to the Creative Commons license, and indicate if changes were made.

\section{REFERENCES}

1. Dowell D, Zhang K, Noonan RK, Hockenberry JM. Mandatory provider review and pain clinic laws reduce the amounts of opioids prescribed and overdose death rates. Health Aff (Millwood). 2016;35:1876-83.

2. Dowell D, Noonan RK, Houry D. Underlying factors in drug overdose deaths. JAMA. 2017;318:2295-6.

3. US Centers for Disease Control and Prevention. Provisional counts of drug overdose deaths as of August 6, 2017. https://www.cdc.gov/nchs/data/ health_policy/monthly-drug-overdose-deathestimates.pdf. Accessed 8 Apr 2018.

4. Guy GP Jr, et al. Vital signs: changes in opioid prescribing in the United States, 2006-2015. MMWR Morb Mortal Wkly Rep. 2017;66(697-704):4.

5. Ingraham C. CDC releases grim new opioid overdose figures: 'We're talking about more than an exponential increase.' The Washington Post, December 21, 2017.

6. Meldrum ML. A capsule history of pain management. JAMA. 2003;290(18):2470-5.

7. Clarke JL, Skoufalos A, Scranton R. The American opioid epidemic: population health implications and potential solutions. Report from the National Stakeholder Panel. Popul Health Manag. 2016;19(1):1-10.

8. Meldrum ML, editor. Opioids and pain relief: a historical perspective. Seattle: International Association for the Study of Pain; 2003.
9. Schiffrin MJ. The management of pain in cancer. St Louis: Year Book; 1956. p. 7-8.

10. Morgan JP. American opiophobia: customary underutilization of opioid analgesics. Adv Alcohol Subst Abuse. 1985;5:163-73.

11. Zenz M, Willweber-Strumpf A. Opiophobia and cancer pain in Europe. Lancet. 1993;341(8852): 1075-6.

12. Marks RM, Sachar EJ. Undertreatment of medical inpatients with narcotic analgesics. Ann Intern Med. 1973;78(2):173-81.

13. Max MB. Improving outcomes of analgesic treatment: is education enough? Ann Intern Med. 1990;113(11):885-9.

14. Porter J, Jick H. Addiction rare in patients treated with narcotics. N Engl J Med. 1980;302(2):123.

15. Portenoy RK, Foley KM. Chronic use of opioid analgesics in non-malignant pain: report of 38 cases. Pain. 1986;25(2):171-86.

16. WHO. Cancer pain relief. Geneva: WHO; 1986.

17. Anderson T. The politics of pain. BMJ. 2010;341: c3800.

18. Melzack R. The tragedy of needless pain. Sci Am. 1990;262(2):27-33.

19. Stein C. Opioid treatment of chronic nonmalignant pain. Anesth Analg. 1997;84(4):912-4.

20. Campbell JN. APS 1995 presidential address. Pain Forum. 1996;1(5):85-8.

21. Mularski RA, White-Chu F, Overbay D, Miller L, Asch SM, Ganzini L. Measuring pain as the 5th vital sign does not improve quality of pain management. J Gen Intern Med. 2006;21(6):607-12.

22. Baker DW. History of the Joint Commission's pain standards: lessons for today's prescription opioid epidemic. JAMA. 2017;317(11):1117-8.

23. Joranson DE, Gilson AM, Dahl JL, Haddox JD. Pain management, controlled substances, and state medical board policy: a decade of change. J Pain Symp Manag. 2002;23(2):138-47.

24. Fenton JJ, Jerant AF, Bertakis KD, Franks P. The cost of satisfaction: a national study of patient satisfaction, health care utilization, expenditures, and mortality. Arch Intern Med. 2012;172(5):405-11.

25. Tucker KL. Medico-legal case report and commentary: inadequate pain management in the context 
of terminal cancer. The case of Lester Tomlinson. Pain Med. 2004;5:214-28.

26. Hwang CS, Chang HY, Alexander GC. Impact of abuse-deterrent OxyContin on prescription opioid utilization. Pharmacoepidemiol Drug Saf. 2015;24(2):197-204

27. Manchikanti L, Kaye AM, Knezevic N, et al. Responsible, safe, and effective prescription of opioids for chronic non-cancer pain: American Society of Interventional Pain Physicians (ASIPP) Guidelines. Pain Physician. 2017;20(2S):S3-92.

28. Frasco PE, Sprung J, Trentman TL. The impact of the Joint Commission for Accreditation of Healthcare Organizations pain initiative on perioperative opiate consumption and recovery room length of stay. Anesth Analg. 2005;100(1):162-8.

29. Vila H Jr, Smith RA, Augustyniak MJ, et al. The efficacy and safety of pain management before and after implementation of hospital-wide pain management standards: is patient safety compromised by treatment based solely on numerical pain ratings? Anesth Analg. 2005;101(2):474-80.

30. Centers for Disease Control and Prevention. National Vital Statistics System mortality data. 2015. http://www.cdc.gov/nchs/deaths.htm. Accessed 8 Apr 2018.

31. Reuters. OxyContin maker Purdue Pharma to stop promoting the drug to doctors. https://www. washingtonpost.com/national/health-science/oxyc ontin-maker-purdue-pharma-to-stop-promoting-thedrug-to-doctors/2018/02/10/c59be118-0ea7-11e8-95 a5-c396801049ef_story.html?utm_term=.bf485594e 8ff. Accessed 8 Apr 2018.

32. The Joint Commission. Pain management. https:// www.jointcommission.org/topics/pain

management.aspx. Accessed 24 April 2017.

33. Gudin J. Oxycodone DETERx ${ }^{\circledR}$ : a novel abuse-deterrent, extended-release analgesic option for the treatment of patients with chronic pain. Pain Ther. 2016;5(2):171-86.

34. Morlion BJ, Mueller-Lissner SA, Vellucci R, et al. Oral prolonged-release oxycodone/naloxone for managing pain and opioid-induced constipation: a review of the evidence. Pain Pract. 2017. https:// doi.org/10.1111/papr.12646.

35. DePriest AZ, Miller K. Oxycodone/naloxone: role in chronic pain management, opioid-induced constipation, and abuse deterrence. Pain Ther. 2014;3(1):1-15.

36. Gudin J, Levy-Cooperman N, Kopecky EA, Fleming $\mathrm{AB}$. Comparing the effect of tampering on the oral pharmacokinetic profiles of two extended-release oxycodone formulations with abuse-deterrent properties. Pain Med. 2015;16(11):2142-51.

37. Jones MR, Carney MJ, Kaye RJ, Prabhakar A, Kaye AD. Drug formulation advances in extended-release medications for pain control. Curr Pain Headache Rep. 2016;20:36.

38. Pergolizzi J, Taylor R, LeQuang J, Raffa R. Managing severe pain and abuse potential: the potential impact of a new abuse-deterrent formulation oxycodone/naltrexone extended-release product. J Pain Res. 2018;11:301-11.

39. Center for Drug Evaluation and Research. Information by Drug Class-Risk Evaluation and Mitigation Strategy (REMS) for opioid analgesics. US Food and Drug Administration home page. https://www. fda.gov/Drugs/DrugSafety/InformationbyDrugClass/ ucm163647.htm. Accessed 8 Apr 2018.

40. Luo ZD. Advancements in pain research. Methods Mol Biol. 2012;851:1-8.

41. "Affordable Care Act." Managed and paid for by the U.S. Centers for Medicare and Medicaid Services, healthcare.gov.

42. Centers for Medicare and Medicaid Services, CMS.gov. The Mental Health Parity and Addiction Equity Act (MHPAEA). www.cms.gov/CCIIO/Programs-and-Initia tives/Other-Insurance-Protections/mhpaea_factsheet. html. Accessed 8 Apr 2018.

43. Krawczyk N, Negron T, Nieto M, Agus D, Fingerhood M. Overcoming medication stigma in peer recovery: a new paradigm. Subs Abus. 2018;12:10.

44. Ringwalt C, Sanford C, Dasgupta N, et al. Community readiness to prevent opioid overdose. Health Promot Pract. 2018. https://doi.org/10. $1177 / 1524839918756887$.

45. Wen L, Warren K. Combating the opioid epidemic: Baltimore's experience and lessons learned. J Public Health (Oxf). 2017;19:1-5.

46. Declaration of Montreal 2010. 2017. http://www. iasp-pain.org/DeclarationofMontreal. Accessed 8 Apr 2018.

47. Blendon RJ, Benson JM. The public and the opioidabuse epidemic. N Engl J Med. 2018;378:407-11.

48. Manchikanti L, Soin A, Mann DP, et al. Reversal of growth of utilization of interventional techniques in managing chronic pain in Medicare population post Affordable Care Act. Pain Phys. 2017;20:551-67.

49. Auriacombe M, Fatséas M, Dubernet J, et al. French field experience with buprenorphine. Am J Addict. 2004;13:S17-28. 